\title{
Serum levels of C1q/tumor necrosis factor-related protein-1 in children with Kawasaki disease
}

\author{
Siqi Feng ${ }^{1}$, Ya Su ${ }^{1}$, Li Luo ${ }^{2}$, Fengchuan Jing $^{2}$ and Qijian $\mathrm{Yi}^{3}$
}

BACKGROUND: To investigate the serum C1q/tumor necrosis factor-related protein-1 (CTRP1) levels in children with acute Kawasaki disease (KD), as well as the relationship between CTRP1 levels and laboratory variables.

METHODS: Eighty-seven children with KD and 38 healthy controls (HCs) were included in this study. General characteristics were obtained from all subjects. Serum CTRP1 levels in all subjects and serum tumor necrosis factor-a (TNF-a), interleukin-1 $\beta$ (IL-1 $\beta$ ), and interleukin-6 (IL-6) levels in KD patients were measured using enzyme-linked immunosorbent assay.

RESULTS: Compared with the HC group, serum CTRP1 levels were significantly elevated in the KD group. Significantly higher serum TNF- $a,\|L-1 \beta\| L-$,6 , and CTRP1 levels were observed in patients with KD with coronary artery lesions (KDCALs) than in patients with KD without CALs (KD-NCALs). Serum CTRP1 levels were positively correlated with white blood cell counts (WBC), percentage of neutrophils (N\%), thrombin time $(T T)$, procalcitonin (Pct), TNF-a, IL-1 $\beta$, and IL-6 levels. Meanwhile, CTRP1 levels were negatively correlated with the percentage of leukomonocytes $(L \%)$ in $\mathrm{KD}$ patients. Furthermore, serum CTRP1 levels were positively correlated with the time point of intravenous immunoglobulin (IVIG), WBC, N\%, TNF-a, IL-1 $\beta$, and IL-6 levels in the KD-CAL group. CONCLUSION: CTRP1 may participate in the process of vasculitis and blood coagulation during the acute phase of KD.

K awasaki disease (KD) was first described in 1967 ((ref. 1)). It is an acute and self-limiting systemic autoimmune vasculitis that primarily affects medium-sized blood vessels, especially coronary arteries. If not treated in a timely manner, $15-25 \%$ children with $\mathrm{KD}$ develop coronary artery lesions (CALs) or coronary artery aneurysms (CAA) with thrombi (2), which can lead to myocardial ischemia, infarction, and even sudden death. Although the exact pathogenesis of $\mathrm{KD}$ has not yet been fully delineated, a number of studies have shown that the immunoreactions in KD result in an increase in inflammatory cytokines that induces vasculitis and the development of CALs $(3,4)$. Our previous study demonstrated that the increased serum levels of inflammatory cytokines, including adiponectin, resistin, tumor necrosis factor- $\alpha$ (TNF- $\alpha$ ), interleukin-1 $\beta$ (IL-1 $\beta$ ), and interleukin-6 (IL-6), participate in the immunoreaction behind vasculitis and are related to the development of CALs in $\operatorname{KD}(5,6)$.

C1q-tumor necrosis factor-related proteins (CTRPs) are part of a highly conserved family of adiponectin paralogs that was recently determined to include the 15 family members of CTRP1-CTRP15 ((ref. 7)). Members of the CTRP family have a modular organization similar to adiponectin and consist of four distinct domains, including an $\mathrm{N}$-terminal signal peptide, a short variable, collagen-like, and C-terminal Clq-like globular domains $(8,9)$. These CTRP family members significantly influence the regulation of glucose and lipid metabolism, inflammation, myocardial protection, and vasodilation(10). CTRP1 is primarily secreted by stromal vascular cells, including adipose tissue macrophages, preadipocytes, and endothelial cells(11). CTRP1 mRNA expression is the highest in adipose tissue $(12,13)$, although it is also expressed in the heart, placenta, muscle, kidneys, prostate, and ovaries (14). Recent evidence demonstrates that increased CTRP1 serum levels are protective against acute ischemic injury in coronary artery diseases(15). TNF- $\alpha$ and IL-1 $\beta$ increase CTRP1 expression(13), and IL-6 expression is positively associated with CTRP1 ((ref. 16)). Furthermore, CTRP1 acts as a potent antithrombotic factor and prevents collageninduced platelet aggregation by blocking von Willebrand's factor from binding to collagen(17).

However, currently, there are no data on serum CTRP1 levels and the relationship between CTRP1 and KDassociated vasculitis. Therefore, we examined the serum CTRP1, TNF- $\alpha$, IL-1 $\beta$, and IL- 6 levels and compared the general inflammatory indices in patients with acute KD.

\section{METHODS}

Subjects and their General Characteristics

Eighty-seven KD patients consisting of 50 males and 37 females (average age $2.373 \pm 0.206$ years) and 38 healthy control (HC) children consisting of 22 males and 16 females (average age

\footnotetext{
${ }^{1}$ Key Laboratory of Pediatrics in Chongqing, Chongqing, China; ${ }^{2}$ Chongqing International Science and Technology Cooperation Center for Child Development and Disorders, Chongqing, China; ${ }^{3}$ Department of Cardiovascular Medicine, Children's Hospital of Chongqing Medical University, Ministry of Education Key Laboratory of Child Development and Disorder, Chongqing, China. Correspondence: Qijian Yi

(1105643760@qq.com)

Received 11 October 2017; accepted 9 January 2018; advance online publication 9 May 2018. doi:10.1038/pr.2018.16
} 


\section{Articles | Feng et al.}

$2.771 \pm 0.304$ years) were enrolled from the Children's Hospital of Chongqing Medical University in Chongqing, China. All of the KD patients were diagnosed in strict accordance with the criteria proposed by the Japanese Circulation Society Joint Working Group(18). The KD subjects were confirmed to be absent of metabolic diseases or any other associated immunological diseases. This study was approved by the Ethics Committee of Children's Hospital, Chongqing Medical University, and written informed consent was obtained from the parents or appropriate guardians of each of the subjects.

Echocardiography was performed on the KD patients within 2 weeks of the onset of the disease and before administration of intravenous immunoglobulin (IVIG) and anticoagulants. The echocardiographic diagnosis met the criteria proposed by the Japanese Kawasaki Disease Research Committee for each of the patients included in this study: CAL was defined by an internal diameter of an artery $\geq 2.5 \mathrm{~mm}$ ( $0-3$ years); $\geq 3.0 \mathrm{~mm}$ ( $3-9$ years); and $\geq 3.5 \mathrm{~mm}$ (9-14 years), or if the internal diameter of a segment was at least 1.5 times that of an adjacent coronary artery; an internal diameter of an artery $>4 \mathrm{~mm}$ was defined as CAA(19). According to echocardiography parameters, $87 \mathrm{KD}$ patients were divided into two groups: KD with CALs (KD-CALs; $n=42$ ) and KD without CALs (KD-NCALs; $n=45)$. All the KD patients were treated with $2 \mathrm{~g} / \mathrm{kg}$ IVIG and oral aspirin after 5 days of fever.

The time point of IVIG, white blood cell counts (WBCs), platelet counts (Plts), hemoglobin ( $\mathrm{Hb})$, percentage of neutrophils (N\%), percentage of leukomonocytes (L\%), mean platelet volume (MPV), platelet distribution width (PDW), plateletcrit (PCT), C-reactive protein (CRP), erythrocyte sedimentation rate (ESR), procalcitonin (Pct), aspartate aminotransferase (AST), alanine aminotransferase (ALT), and creatine kinase-MB (CK-MB), as well as the blood coagulation parameters prothrombin time (PT), PT international normalized ratio (PT-INR), activated partial thromboplastin time (APTT), fibrinogen (FIB), thrombin time (TT), and D-dimer (DD) were collected from all KD patients. Blood samples were drawn before administration of IVIG and anticoagulants.

\section{Sample Collection and Processing}

Venous blood samples were drawn from the KD patients on the day of admission before receiving IVIG and anticoagulants. The blood samples were then immediately centrifuged at 3,000 revolutions/min for $10 \mathrm{~min}$ to separate out the serum. The same procedure was performed to obtain serum samples from the HCs. All the blood samples were stored at $-80^{\circ} \mathrm{C}$.

\section{Measurement of Serum CTRP1, TNF- $\alpha$, IL-1 $\beta$, and IL- 6 Concentrations}

Serum levels of CTRP1 in all subjects and TNF- $\alpha$, IL-1 $\beta$, and IL- 6 levels in $\mathrm{KD}$ patients were measured using an enzyme-linked immunosorbent assay kit (RayBiotech, Atlanta, GA) according to the manufacturer's instructions. All the samples were measured in duplicate at the appropriate dilutions. The intra- and interassay coefficients of variation for the levels of CTRP1, TNF- $\alpha$, IL- $1 \beta$, and IL- 6 were $<10 \%$ and $<12 \%$, respectively.

\section{Statistical Analysis}

Serum cytokine levels were analyzed as quantitative traits. All the values are described as mean \pm SD or number and percent $(n, \%)$. Comparisons of frequencies between groups were analyzed using $\chi^{2}$ test. Differences between groups were assessed using Student's $t$-tests. Pearson's correlation was used for analysis of associations between sequential parameters. SPSS 19.0 software for Windows (SPSS, Chicago, IL) was used for all statistical analyses. A two-sided $P$ value of less than 0.05 was considered statistically significant.

\section{RESULTS}

\section{Serum CTRP1 Levels in all Study Subjects}

When comparing the $\mathrm{KD}$ and $\mathrm{HC}$ groups, no significant differences in age and gender $(P>0.05)$ were found. As

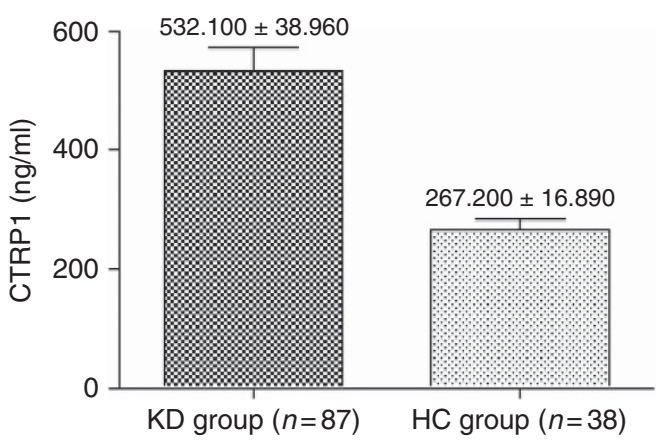

Figure 1. Serum CTRP1 levels in KD patients and healthy controls. CTRP1, C1q/tumor necrosis factor-related protein-1; KD, Kawasaki disease.

Table 1. General characteristics and serum TNF- $\alpha, I L-1 \beta, I L-6$, and CTRP1 levels in KD-CAL and KD-NCAL groups

\begin{tabular}{|c|c|c|c|}
\hline & KD-CALs $(n=42)$ & KD-NCALs $(n=45)$ & $P$ value \\
\hline $\begin{array}{l}\text { Age at diagnosis } \\
(\mathrm{Yr})\end{array}$ & $2.201 \pm 0.254$ & $2.533 \pm 0.321$ & 0.424 \\
\hline $\begin{array}{l}\text { Gender (male/ } \\
\text { female) }\end{array}$ & $28 / 14$ & $22 / 23$ & 0.096 \\
\hline $\begin{array}{l}\text { Time point of } \\
\text { IVIG (day) }\end{array}$ & $7.133 \pm 0.420$ & $6.429 \pm 0.237$ & 0.155 \\
\hline WBC $\left(10^{3} / \mu \mathrm{l}\right)$ & $16.570 \pm 1.032$ & $16.460 \pm 0.859$ & 0.935 \\
\hline Plt $\left(10^{3} / \mu \mathrm{l}\right)$ & $381.100 \pm 20.570$ & $407.900 \pm 25.780$ & 0.423 \\
\hline $\mathrm{Hb}(\mathrm{g} / \mathrm{l})$ & $105.600 \pm 1.548$ & $109.300 \pm 1.655$ & 0.110 \\
\hline N\% & $0.699 \pm 0.018$ & $0.649 \pm 0.285$ & 0.151 \\
\hline L\% & $0.241 \pm 0.157$ & $0.291 \pm 0.025$ & 0.106 \\
\hline MPV (fl) & $9.533 \pm 0.160$ & $9.618 \pm 0.162$ & 0.712 \\
\hline PDW (fl) & $10.780 \pm 0.304$ & $10.680 \pm 0.260$ & 0.796 \\
\hline PCT (\%) & $0.411 \pm 0.024$ & $0.437 \pm 0.023$ & 0.432 \\
\hline CRP (mg/dl) & $57.390 \pm 6.600$ & $65.910 \pm 6.917$ & 0.377 \\
\hline $\mathrm{ESR}(\mathrm{mm} / \mathrm{h})$ & $65.600 \pm 3.468$ & $74.730 \pm 3.991$ & 0.090 \\
\hline Pct (ng/ml) & $2.184 \pm 0.685$ & $1.060 \pm 0.283$ & 0.124 \\
\hline AST (U/I) & $39.510 \pm 4.759$ & $39.200 \pm 4.263$ & 0.961 \\
\hline $\mathrm{ALT}(\mathrm{U} / \mathrm{l})$ & $52.140 \pm 8.502$ & $57.250 \pm 10.630$ & 0.725 \\
\hline CK-MB (U/I) & $0.903 \pm 0.222$ & $0.703 \pm 0.156$ & 0.458 \\
\hline TNF-a (pg/ml) & $1,306.000 \pm 109.600$ & $417.300 \pm 34.500$ & $<0.0001^{*}$ \\
\hline $\mathrm{IL}-1 \beta(\mathrm{pg} / \mathrm{ml})$ & $30.380 \pm 2.433$ & $7.225 \pm 0.849$ & $0.001^{*}$ \\
\hline IL-6 (pg/ml) & $257.200 \pm 24.030$ & $165.500 \pm 19.220$ & $0.004^{*}$ \\
\hline CTRP1 (ng/ml) & $668.900 \pm 71.400$ & $404.300 \pm 23.100$ & $0.001^{*}$ \\
\hline
\end{tabular}

ALT, alanine aminotransferase; AST, aspartate aminotransferase; CAL, coronary artery lesion; CK-MB, creatine kinase-MB; CRP, C-reactive protein; CTRP1, C1q/tumor necrosis factor-related protein-1; ESR, erythrocyte sedimentation rate; IL-1 $\beta$, interleukin-1 $\beta$; IL-6, interleukin-6; KD, Kawasaki disease; L\%, percentage of leukomonocytes; MPV, mean platelet volume; N\%, percentage of neutrophils; NCAL, non-CAL; Pct, procalcitonin; PCT, plateletcrit; PDW, platelet distribution width; PIt, platelet count; TNF$a$, tumor necrosis factor-a; WBC, white blood cell count; Yr, year. ${ }^{*} P<0.05$.

shown in Figure 1, compared with the HC group, serum CTRP1 levels were significantly higher in the KD group $(P<0.05)$. 
Laboratory Variables and Serum TNF- $\alpha$, IL-1 $\beta$, IL-6, and CTRP1 Levels in the KD-CAL and KD-NCAL Groups

Upon comparison, no significant differences in age, gender, the time point of IVIG, WBC, Plt, Hb, N\%, L\%, MPV, PDW, PCT, CRP, ESR, Pct, AST, ALT, or CK-MB were discerned between the KD-CAL and KD-NCAL groups $(P>0.05)$. However, as presented in Table 1, significant increases in serum TNF- $\alpha$, IL-1 $\beta$, IL- 6 , and CTRP1 levels were found in the KD-CAL group compared with the KD-NCAL group $(P<0.05)$.

\section{Correlations Between CTRP1 Levels and Laboratory Variables in all KD Patients}

Serum CTRP1 levels in KD patients were not significantly correlated with the time point of IVIG, Plt, Hb, MPV, PDW, PCT, CRP, ESR, AST, ALT, or CK-MB $(P>0.05)$. However, serum CTRP1 levels were positively correlated with WBC, N\%, Pct, TNF- $\alpha$, IL-1 $\beta$, and IL-6 levels $(p<0.05)$, and were negatively correlated with $\mathrm{L} \%(p<0.05)$ in patients with $\mathrm{KD}$ (Table 2).

\section{Correlations Between CTRP1 Levels and Laboratory Variables in the KD-CAL and KD-NCAL Groups}

There were no significant associations identified between serum CTRP1 levels and the laboratory variables, including

Table 2. Correlations between CTRP1 levels and laboratorymeasured variables in all KD patients

\begin{tabular}{|c|c|c|}
\hline & \multicolumn{2}{|c|}{ CTRP1 } \\
\hline & $r$ & $P$ \\
\hline Time point of IVIG (day) & 0.126 & 0.244 \\
\hline WBC $\left(10^{3} / \mu \mathrm{l}\right)$ & 0.289 & $0.007^{*}$ \\
\hline Plt $\left(10^{3} / \mu \mathrm{l}\right)$ & -0.007 & 0.953 \\
\hline $\mathrm{Hb}(\mathrm{g} / \mathrm{l})$ & -0.154 & 0.154 \\
\hline $\mathrm{N} \%$ & 0.257 & $0.016^{*}$ \\
\hline L\% & -0.244 & $0.023^{*}$ \\
\hline MPV (fl) & -0.128 & 0.236 \\
\hline PDW (fl) & -0.116 & 0.286 \\
\hline PCT (\%) & -0.143 & 0.186 \\
\hline CRP (mg/dl) & 0.003 & 0.982 \\
\hline $\operatorname{ESR}(\mathrm{mm} / \mathrm{h})$ & 0.005 & 0.964 \\
\hline Pct (ng/ml) & 0.615 & $<0.0001^{*}$ \\
\hline AST (U/I) & 0.067 & 0.537 \\
\hline $\operatorname{ALT}(\mathrm{U} / \mathrm{I})$ & 0.117 & 0.280 \\
\hline CK-MB (U/I) & -0.012 & 0.909 \\
\hline TNF-a (pg/ml) & 0.596 & $<0.0001^{*}$ \\
\hline IL-1 $\beta(p g / m l)$ & 0.677 & $<0.0001^{*}$ \\
\hline IL-6 (pg/ml) & 0.324 & $0.002^{*}$ \\
\hline $\begin{array}{l}\text { ALT, alanine aminotransferas } \\
\text { kinase-MB; CRP, C-reactive pr } \\
\text { tein-1; ESR, erythrocyte sedin } \\
\text { IL-6, interleukin-6; KD, Kawas } \\
\text { mean platelet volume; N\%, } \\
\text { calcitonin; PDW, platelet distri } \\
\text { sis factor-a; WBC, white blood } \\
{ }^{*} P<0.05 \text {. }\end{array}$ & $\begin{array}{l}\text { ate aminot } \\
\mathrm{Cla} / \text { tumor } \\
\mathrm{Hb} \text {, hemo o } \\
\text { b, percentas } \\
\text { neutrophils } \\
\text { Plt, platelet } \\
\text { year. }\end{array}$ & $\begin{array}{l}\text { MB, creatine } \\
\text {-related pro- } \\
\text { terleukin-1 } \beta \text {; } \\
\text { nocyte; MPV, } \\
\text { crit; Pct, pro- } \\
\text { tumor necro- }\end{array}$ \\
\hline
\end{tabular}

the time point of IVIG, WBC, Plt, Hb, N\%, L\%, MPV, PDW, PCT, CRP, ESR, AST, ALT, CK-MB, and Pct, TNF- $\alpha$, IL- $1 \beta$, and IL-6 levels in the KD-NCALs group $(P>0.05)$. However, serum CTRP1 levels were significantly positively correlated with the time point of IVIG, WBC, N\%, and Pct, TNF- $\alpha$,

Table 3. Correlations between CTRP1 levels and laboratorymeasured variables in KD-CAL and KD-NCAL patients

\begin{tabular}{|c|c|c|c|c|}
\hline & \multicolumn{2}{|c|}{$\mathrm{KD}-\mathrm{CAL}(n=42)$} & \multicolumn{2}{|c|}{ KD-NCAL $(n=45)$} \\
\hline & $r$ & $P$ & $r$ & $P$ \\
\hline Time point of IVIG (day) & 0.455 & $0.002^{*}$ & -0.079 & 0.604 \\
\hline WBC $\left(10^{3} / \mu \mathrm{l}\right)$ & 0.397 & $0.009^{*}$ & 0.076 & 0.619 \\
\hline Plt $\left(10^{3} / \mu \mathrm{l}\right)$ & 0.122 & 0.441 & -0.037 & 0.810 \\
\hline $\mathrm{Hb}(\mathrm{g} / \mathrm{l})$ & -0.223 & 0.155 & 0.022 & 0.886 \\
\hline N\% & 0.302 & $0.048^{*}$ & 0.057 & 0.712 \\
\hline L\% & -0.262 & 0.094 & -0.003 & 0.829 \\
\hline MPV (fl) & 0.016 & 0.919 & 0.041 & 0.789 \\
\hline PDW (fl) & -0.009 & 0.953 & 0.087 & 0.569 \\
\hline PCT (\%) & 0.016 & 0.920 & -0.135 & 0.375 \\
\hline CRP (mg/dl) & 0.082 & 0.607 & 0.071 & 0.643 \\
\hline ESR $(\mathrm{mm} / \mathrm{h})$ & 0.025 & 0.873 & -0.174 & 0.252 \\
\hline Pct (ng/ml) & 0.434 & $0.004^{*}$ & 0.281 & 0.061 \\
\hline AST (U/I) & 0.236 & 0.132 & 0.233 & 0.123 \\
\hline ALT (U/I) & 0.160 & 0.313 & 0.008 & 0.959 \\
\hline CK-MB (U/I) & -0.005 & 0.975 & -0.140 & 0.359 \\
\hline TNF-a (pg/ml) & 0.593 & $<0.0001^{*}$ & 0.198 & 0.083 \\
\hline IL-1 $\beta$ (pg/ml) & 0.534 & $<0.0001^{*}$ & 0.020 & 0.909 \\
\hline IL-6 (pg/ml) & 0.421 & $<0.0001^{*}$ & 0.267 & 0.077 \\
\hline
\end{tabular}

ALT, alanine aminotransferase; AST, aspartate aminotransferase; CAL, coronary artery lesion; CK-MB, creatine kinase-MB; CRP, C-reactive protein; CTRP1, C1q/tumor necrosis factor-related protein-1; ESR, erythrocyte sedimentation rate; $\mathrm{Hb}$, hemoglobin; IL$-1 \beta$, interleukin-1 $\beta$; IL-6, interleukin-6; IVIG, intravenous immunoglobulin; KD, Kawasaki disease; L\%, percentage of leukomonocytes; MPV, mean platelet volume; $\mathrm{N} \%$, percentage of neutrophils; NCAL, non-CAL; PCt, procalcitonin; $\mathrm{PCT}$, plateletcrit; PDW, platelet distribution width; TNF-a, tumor necrosis factor-a; WBC, white blood cell. ${ }^{*} P<0.05$.

Table 4. Correlations between CTRP1 levels and laboratorymeasured variables in KD-CAL and KD-NCAL patients

\begin{tabular}{lcc}
\hline & \multicolumn{2}{c}{ CTRP1 } \\
\cline { 2 - 3 } & $r$ & $P$ \\
\hline PT (s) & -0.172 & 0.338 \\
PT-INR & -0.157 & 0.382 \\
APTT (s) & -0.036 & 0.842 \\
FIB (g/l) & -0.001 & 0.996 \\
TT (s) & 0.345 & $0.049^{*}$ \\
DD (mg/l) & -0.072 & 0.689 \\
\hline
\end{tabular}

APTT, activated partial thromboplastin time; CAL, coronary artery lesion; CTRP1, C1q/tumor necrosis factor-related protein-1; DD, D-dimer; FIB, fibrinogen; KD, Kawasaki disease; PT, prothrombin time; PT-INR, PT international normalized ratio; s, second; $\pi$, thrombin time.

${ }^{*} P<0.05$. 
IL-1 $\beta$, and IL-6 levels $(P<0.05)$ in the KD-CALs group (Table 3).

\section{Correlation Between CTRP1 Levels and Blood Coagulation Parameters in KD Patients}

Serum CTRP1 levels were not significantly correlated with PT, PT-INR, APTT, FIB, or DD $(P>0.05)$. However, there was a positive correlation between the CTRP1 levels and TT $(P<0.05$; Table 4).

\section{DISCUSSION}

CTRP1 has been reported to regulate metabolism $(20,21)$ and its expression is induced during inflammation(11). Inflammatory cytokines such as TNF- $\alpha$ and IL- $1 \beta$ could mediate the elevations of CTRP1 ((ref. 13)). We were motivated to study serum CTRP1 levels in terms of the relationship between serum CTRP1 levels and vasculitis and CALs in children with $\mathrm{KD}$. We found that serum CTRP1 levels in acute KD patients were significantly higher than those in the HCs, and serum CTRP1, TNF- $\alpha$, IL- $1 \beta$, and IL- 6 concentrations were even higher in the KD-CAL group than in the KD-NCAL group. Furthermore, we found that CTRP1 levels were positively correlated with WBC, N\%, TT, Pct, TNF- $\alpha$, IL- $1 \beta$, and IL-6 levels, but were negatively correlated with L\% in patients with $\mathrm{KD}$. In addition, serum CTRP1 levels were positively correlated with the time point of IVIG, the levels of WBC, N\%, Pct, TNF- $\alpha$, IL-1 $\beta$, and IL- 6 in the KDCALs group.

As an inflammatory factor, CTRP1 was found to act in many chronic diseases such as coronary artery diseases(22), and there were no data about CTRP1 levels in acute diseases such as KD. Our present study found that CTRP1 levels were much higher in acute KD patients than in the HC group, indicating that CTRP1 may act as an inflammatory factor in the vasculitis in the acute phase of $\mathrm{KD}$. Furthermore, the CTRP1, TNF- $\alpha$, IL- $1 \beta$, and IL-6 levels in the KD-CAL group were much higher than in the KD-NCAL group. As our previous study demonstrates, the increased TNF- $\alpha$, IL- $1 \beta$, and IL-6 levels participate in the inflammation associated with the development of CALs. Similarly, the increased CTRP1 levels in KD-CALs revealed that CTRP1 may mediate the development of CALs in KD. As reported, inflammatory cytokines such as TNF- $\alpha$, IL- $1 \beta$, and IL- 6 could promote the elevations of CTRP1 expression, and the increased levels of TNF- $\alpha$, IL-1 $\beta$, and IL-6 in KD may induce the CTRP1 expression to regulate the vasculitis. In addition, we found that the CTRP1 levels were positively correlated with $\mathrm{WBC}, \mathrm{N} \%$, and Pct levels and negatively correlated with $\mathrm{L} \%$ in $\mathrm{KD}$ patients. Studies have indicated that bacterial or viral infections participate in the pathogenesis of $\operatorname{KD}(23,24)$. Bacteria and viruses have been implicated as the causative agents of $\mathrm{KD}$ (25). As indices of infections, WBC, N\%, and Pct levels reflect the inflammation in KD. High WBC, N\%, L\%, and Pct values indicate serious inflammation. When inflammation occurs, CTRP1 levels increase to regulate the inflammation, and our data indicate that CTRP1 acts as an inflammatory cytokine in
KD. However, as WBC, N\%, and Pct are typically significantly elevated and $\mathrm{L} \%$ decreased when the body has a bacterial infection, CTRP1 levels may be more sensitive to bacterial infections than viral infections. Kim et al. (13) found that TNF- $\alpha$ and IL- $1 \beta$ increase lipopolysaccharide-induced CTRP1 expression in adipose tissue. The study by Tang and coworkers revealed that CTRP1 expression is positively correlated with IL-6 levels(17). We obtained similar results in this study, where TNF- $\alpha$, IL- $1 \beta$, and IL- 6 levels were positively correlated with CTRP1 levels. These cytokines were increased to induce the secretion of CTRP1. Furthermore, serum CTRP1 levels were positively correlated with WBC, Plt, N\%, Pct, TNF- $\alpha$, IL- $1 \beta$, and IL- 6 levels. With the more severe inflammation in KD with CALs, CTRP1 may be highly secreted and promote the inflammatory factors during the vasculitis in $\mathrm{KD}$. The pathological process behind $\mathrm{KD}$ is complex and much of it remains unknown. Serum cytokines, such as TNF- $\alpha$, IL-1 $\beta$, IL-6, and CTRP1, may act synergistically to exert a positive effect on the development of vasculitis. $\mathrm{KD}$ is invariably associated with an inflammatory process, with an elevation in WBC, CRP, and $\operatorname{ESR}(26)$. Typically, WBC, CRP, and ESR values are regarded as predictors of the levels of inflammation and the occurrence of CAA. However, in this study, serum CTRP1 levels were not significantly associated with CRP or ESR in either KD patients or the subgroups of $\mathrm{KD}-\mathrm{CAL}$ and $\mathrm{KD}-\mathrm{NCAL}$ patients, suggesting that CTRP1, CRP, and ESR may act through different signaling pathways during pathological processes.

Randomized controlled trials and meta-analysis have unequivocally demonstrated the administration of IVIG and aspirin reduces the occurrence of CAA(27). The optimal dose of IVIG is $2 \mathrm{~g} / \mathrm{kg}$, usually administered as a single infusion. If the IVIG is not administered in a timely manner, a percentage of children with $\mathrm{KD}$ will develop CALs(2). In this study, the time point of IVIG in KD-CALs was a little longer than that in KD-NCALs. Although the difference was not significant, the time point of IVIG correlated with the increased incidence of CALs. In addition, serum CTRP1 levels were significantly correlated with the time point of IVIG in the KD-CAL group. As the period of time between the illness day and starting IVIG increased, the vasculitis became more serious and more endothelial cells and smooth muscle cells were injured, thus aiding in the development of CALs. Under inflammatory stress, CTRP1 may increase to mediate KD-associated vasculitis.

Via its C-terminal C1q-like globular domain, CTRP1 could inhibit collagen-induced platelet aggregation by blocking von Willebrand's factor binding to collagen(18). In our study, we found that the TT in KD-CAL group was longer than that in the KD-NCAL group (data not shown); however, this difference was not statistically significant. However, CTRP1 concentrations were positively correlated with TT. These data indicate that CTRP1 may positively regulate blood coagulation and prevent thrombus formation in $\mathrm{KD}$. 


\section{CONCLUSION}

This is the first study to demonstrate that serum CTRP1 levels are significantly elevated in $\mathrm{KD}$ children with CALs. The serum TNF- $\alpha$, IL- $1 \beta$, and IL- 6 levels correlated with serum CTRP1 levels during the acute phase of KD. These observations indicate that CTRP1 has an indispensable role in KD-associated vasculitis, and CTRP1, TNF- $\alpha$, IL- $1 \beta$, and IL-6 may exert synergetic effects on the regulation of inflammation during the acute phase of KD. Furthermore, CTRP1 may positively regulate coagulation of the blood and prevent thrombus formation in $\mathrm{KD}$ patients. As the volunteers are limited, the HCs are in a lower proportion than patients with KD. The mechanism of action and signaling pathway(s) involved in the role of CTRP1 in vasculitis and development of CALs in acute KD remain to be further investigated.

\section{ACKNOWLEDGMENTS}

We acknowledge Hu Dou in Children's Hospital of Chongqing Medical University for his help in collecting consents from patients and receiving the samples and we thank all children enrolled in the study for their kindness. Ethical approval: The study protocol was approved by the Ethics Committee of Children's Hospital of Chongqing Medicine University, and written informed consent forms were obtained from the parents or other guardians of all subjects.

Disclosure: The authors declare no conflict of interest.

\section{REFERENCES}

1. Kawasaki T. Acute febrile mucocutaneous syndrome with lymphiod involvement with specific desquamation of the fingers and toes in children. Arerugi 1967;16:178-222.

2. Kato H, Ichinose E, Yoshioka F, et al. Fate of coronary aneurysms in Kawasaki disease: serial coronary angiography and long-term followup study. Am J Cardiol 1982;49:1758-66.

3. Gitiaux C, Kossorotoff M, Bergounioux J, et al. Cerebralvasculitis in severe Kawasaki disease: early detection by magneticresonance imaging and good outcome after intensivetreatment. Dev Med Child Neurol 2012;54:1160-3.

4. Gerding R. Kawasaki disease: a review. J Pediatr Health Care 2011;25: 379-87.

5. Liu R, He B, Gao F, et al. Relationship between adipokines and coronary artery aneurysm in children with Kawasaki disease. Transl Res 2012;160: 131-6.

6. Si F, Wu Y, Gao F, Feng S, et al. Relationship between IL-27 and coronary arterial lesions in children with Kawasaki disease. Clin Exp Med 2017;17: $451-7$.

7. Seldin MM, Peterson JM, Byerly MS, et al. Myonectin (CTRP15), a novel myokine that links skeletal muscle to systemic lipid homeostasis. J Biol Chem 2012;287:11968-80.

8. Ahima RS, Qi Y, Singhal NS, et al. Brain adipocytokine action and metabolic regulation. Diabetes 2006;55:145-54.

9. Scherer PE, Williams S, Fogliano M, et al. A novelserum protein similar to C1q, produced exclusively in adipocytes. J Biol Chem 1995;270: 26746-9.
10. Wang H, Wang R, Du D, et al. Serum levels of C1q/TNF-related protein1 (CTRP1) are closely associated with coronary artery disease. BMC Cardiovasc Disord 2016;16:92.

11. Schäffler A, Buechler C. CTRP family: linking immunity to metabolism. Trends Endocrinol Metab 2012;23:194-204.

12. Wong GW, Krawczyk SA, Kitidis-Mitrokostas C, et al. Molecular, biochemical and functional characterizations of C1q/TNF family members: adipose-tissue-selective expression patterns, regulation by PPAR-gamma agonist, cysteine-mediated oligomerizations, combinatorial associations and metabolic functions. Biochem J 2008;416:161-77.

13. Kim KY, Kim HY, Kim JH, et al. Tumor necrosis factor-alpha and interleukin-1beta increases CTRP1 expression in adipose tissue. FEBS Lett 2006;580:3953-60.

14. Innamorati G, Whang MI, Molteni R, et al. GIP, a G-protein-coupled receptor interacting protein. Regul Pept 2002;109:173-9.

15. Yuasa D, Ohash K, Shibata R, et al. C1q-TNF-related protein functions to protect against acute ischemic injury in the heart. FASEB J 2016;30: 1065-75.

16. Tang JN, Shen BL, Lin CL, et al. Plasma levels of C1q/TNF-related protein 1 and interleukin 6 in patients with acute coronary syndrome or stable angina pectoris. Am J Med Sci 2015;349:130-6.

17. Lasser G, Guchhait P, Ellsworth JL, et al. C1qTNF-related protein-1 (CTRP-1): a vascular wallprotein that inhibits collagen-induced platelet aggregation by blocking VWF binding to collagen. Blood 2006;107: 423-30.

18. Japanese Circulation Society Joint Working Group. Guidelines for diagnosis and management of cardiovascular sequelae in Kawasaki disease (JCS2013). Circ J 2014;78:2521-62.

19. Ayusuwa M, Sonobe T, Uemura S, et al. Revision of diagnostic guidlines for Kawasaki disease (the 5th revised edition). Pediatr Int 2005;47:232-4.

20. Pan X, Lu F, Wu F, et al. Circulating complement-C1q TNF-related protein 1 levels are increased in patients with type 2 diabetes and are associated with insulin sensitivity in Chinese subjects. PLoS ONE 2014;9: e94478.

21. Chalupova L, Zakovska A, Adamcova K. Development of a novel enzyme-linked immunosorbent aassay (ELISA) for measurement of serum CTRP1: a pilot study: measurement of serum CTRP1 in healthy donors and patients with metabolic syndrome. Clin Biochem 2013;46: 73-8.

22. Lu L, Zhang RY, Wang XQ, et al. C1q/TNF-related protein-1: an adipokine marking and promoting atherosclerosis. Eur Heart J 2016;37: 23.

23. Neuburger JW, Takahaski M, Gerber MA, et al. Diagnosis, treatment, and long-term management of Kawasaki disease: a statement for health professionals from the Committee on Rheumatic Fever, Endocarditis, and Kawasaki Disease, Council on Cardiovascular Disease in the Young, American Heart Association. Pediatrics 2004;114:1708-33.

24. Komatsu H, Fujisawa T. Kawasaki disease and infection. Nihon Rinsho. 2008;66:278-82.

25. Hiromichi H, Tsuyoshi S, Kunihiro O. Comprehensive pathogen detectin associated with four recurrent episodes of Kawasaki disease in a patient during a single year using next-generation sequencing. JMM Case Rep 2016;3:e005019.

26. Eleftheriou D, Levin M, Shingadia D, et al. Management of Kawasaki disease. Arch Dis Child 2014;99:74-83.

27. Donato R, A Laura, F Michele, et al. Critical overview of the risk scoring systems to predict non-responsiveness to intravenous immunoglobulin in Kawasaki syndrome. Int J Mol Sci 2016;17:278. 